\title{
PLURALIZANDO LA TEOLOGíA POLÍTiCA NUEVAS AGENDAS EN TORNO A UN ANTIGUO PROBLEMA
}

\author{
Diego Rossello ${ }^{1}$ \\ Universidad Adolfo Ibáñez, Chile
}

“...hay mucha más teología política en la vida cotidiana

de lo que jamás hemos pensado"

Eric Santner, The Royal Remains: The People's Two Bodies and

the Endgames of Sovereignty

${ }^{1}$ Diego Rossello es Doctor en Ciencia Política, con especialización en filosofía política, por Northwestern University, Estados Unidos. Se desempeña como Profesor Asociado en el Departamento de Filosofía, Facultad de Artes Liberales, Universidad Adolfo Ibáñez. El presente trabajo es parte del Proyecto FONDECYT Regular Nro. 1171154. 
Cuarenta años atrás la teología política era un área de estudio marginal circunscrita a un grupo pequeño de especialistas eruditos. En esos tiempos, la teología política entendida como tematización sistemática del vínculo entre lo teológico y lo político se perfilaba como una corriente menor al interior de la investigación académica más establecida sobre el problema de la secularización y la filosofía de la historia. ${ }^{2}$ Hoy, sin embargo, la teología política se presenta como un programa de investigación vibrante y en expansión dentro de la filosofía política, tanto en lo que concierne a las temáticas que aborda como a períodos históricos y tradiciones religiosas --y no religiosas-- que incluye en su análisis. ${ }^{3}$

En este sentido, por un lado, la teología política se ha extendido más allá de los trabajos clásicos de Baruch Spinoza, Carl Schmitt, Walter Benjamin, Leo Strauss y Ernst Kantorowicz, ${ }^{4}$ todos ellos ubicados al interior de la tradición judeo-cristiana, para expandirse hacia la pluralidad de tradiciones religiosas que Jaspers incluyó en la era axial ${ }^{5}$ e incursionar, asimismo, en espacios de saber heterodoxos como los estudios de animalidad y los nuevos materialismos, entre otros. ${ }^{6}$ Como es de esperarse, esta expansión trae consigo nuevos riesgos y desafíos, entre ellos el problema de la especificidad y el estiramiento conceptual. Con todo, la teología política atraviesa un proceso de pluralización creciente ${ }^{7}$ que, sugerimos, constituye un aporte a la problematización de una serie de dualismos estancos entre lo secular y lo religioso, lo profano y lo sagrado, la razón y la revelación, la ciencia y la fe. En consecuencia, el presente dossier pretende hacer lugar a la pluralización que atraviesa la teología política incluyendo para ello aportes no sólo desde el cristianismo y el judaísmo, sino también desde la tradición musulmana, el feminismo, el ecocriticismo y el ateísmo.

${ }^{2}$ Al efecto ver Weber (1958); Schmitt (2006); Adorno y Horkheimer (2007); Löwith (1957); Bluemenberg (1985).

${ }^{3}$ Peterson (1999); Maritain (2002); Rosenzweig (2005); Girard (1979); Santner (2001, 2006 y 2011); Lefort (2006); Agamben (1998, 2005 y 2011); Schwartz (2008); Derrida (2001); Kahn (2016); Lupton (2014); Mahmood (2011); Assmann (2008 y 2018); Hägglund (2019); Fenves (2010); Martel (2014); Meier (2006); Asad (2003); Dotti (1996 y 2002); Esposito (2015); Zizek y Millbank (2011); Hammill y Lupton (2012); Laclau (2006).

${ }^{4}$ Spinoza (2001); Schmitt (2006); Benjamin (1999); Strauss (1996); Kantorowicz (1997).

${ }^{5}$ Véase Jaspers (1953). Se utiliza el concepto de la era axial solo de manera descriptiva, a sabiendas de que se trata de un mito o atajo conceptual más que de una descripción acabada de un período histórico coherente.

${ }^{6}$ Linzey (1994 y 2009); Moore (2014); Dean-Drummond and Clough (2009); Meyer (2018); Keller and Rubenstein (2018); Cheng (2001).

${ }^{7}$ Connolly (1995) y Connolly (2000). 
Pero un/a lector/a escéptico podría sugerir, con buen tino, que incluso si se aceptase que la teología política puede hacer un aporte a la clarificación de los dualismos mencionados aún queda por justificar en qué medida aquella sería relevante en un mundo post-secular, y a qué nos referimos con esta última expresión. En su importante libro A Secular Age, Charles Taylor se opone a las que denomina "historias de sustracción" según las cuales la modernidad occidental produciría una separación tajante entre iglesia y estado y un declive gradual en la creencia religiosa. Frente a estas dos maneras de entender la modernidad secular, Taylor agrega una tercera que indica que "la creencia en Dios ya no es axiomática. Existen otras opciones" (Taylor 2007: 3), lo cual lo lleva a concluir que la creencia religiosa atraviesa una transformación desafiante, pero que de ella no se sigue sin más un declive ni una desaparición progresiva de la religión. Más bien, Taylor propone que la creencia en Dios entendida como una opción entre varias ofrece nuevos escenarios y posibilidades para la religión. Si se complementa el trabajo de Taylor, focalizado principalmente en la secularización europea y nordatlántica, con nuevos desarrollos sobre formaciones de lo secular ${ }^{8}$ y variedades de secularismo 9 en el mundo, el panorama se complejiza bastante. De este modo, las sociedades occidentales presentan una concepción de secularismo entre muchas posibles (y con vastas diferencias en su interior: no es lo mismo la laicidad francesa que el secularismo inglés o norteamericano) por lo que sería fútil, y en última instancia inconducente, intentar imponer universalmente una concepción semejante a otras tradiciones religiosas y culturales como el Islam, el Budismo, el Hinduismo e incluso el Judaísmo. ${ }^{10}$

Por ello, la pluralidad de formaciones y variedades de lo secular, así como el hecho de que la religión ha devenido una opción entre muchos otros proyectos de vida posibles, invita a re-conceptualizar la época en la que nos encontramos más allá del dualismo entre lo secular y lo religioso como una época post-secular. En este sentido, tanto Jürgen Habermas como José Casanova han propuesto que a pesar de que la religión se ha retirado a la esfera de lo privado, y se ha circunscrito a un rol de "cuidado pastoral", ello no implica que haya desaparecido ni que esté en vías de desaparición (Habermas 2009: 63; Casanova 1994). Por el contrario, si la modernización secular europea hace décadas se percibía a sí misma como un faro que irradiaba la luz de la razón hacia los rincones más recónditos del planeta, hoy se muestra como una excepción idiosincrásica cuestionada y tensionada doblemente: desde el interior, por escenarios post-coloniales que traen nuevos inmigrantes creyentes no cristianos a la metrópoli; desde el exterior, por superpotencias mayoritariamente cristianas como los Estados Unidos y por nuevos factores de poder internacional (China, Rusia, Turquía, India, Brasil, el

\footnotetext{
${ }^{8}$ Asad (2003).

${ }^{9}$ Warner (2013).

${ }^{10}$ Asad (2018), Mahmood (2016); Singh (2018); Clooney (2003).
} 
mundo Islámico) que no siguen, ni parecen querer seguir, un modelo de secularización al estilo europeo.

Aunque no puede hacerlo de manera exhaustiva, el presente dossier pretende ofrecer un panorama de las diversas maneras de hacer teología política hoy. Para ello incluye contribuciones que tematizan legados cristianos, judaicos, musulmanes y ateos, en los géneros de la filosofía y la teoría política, la historia del pensamiento político, la genealogía de los conceptos, el feminismo y el eco-criticismo, entre otros. Al hacerlo, también presupone y reconoce la presencia extendida de la cuestión de la teología política en el contexto de la discusión filosófica latinoamericana, especialmente en el cono sur. ${ }^{11}$ Con el objetivo de captar esta diversidad de abordajes, temas y énfasis, el presente dossier reúne trabajos de académicos/as que enseñan e investigan sobre teología política en universidades de Chile, Argentina, México, España, Australia y Estados Unidos. En lo que sigue, entonces, presentamos brevemente el contenido de cada uno de los artículos que conforman el dossier.

El trabajo de Montserrat Herrero López, profesora de la Universidad de Navarra (España), interpreta el pensamiento del reconocido teórico político argentino Ernesto Laclau, creador del post-marxismo, como una teología política revolucionaria. Por su parte, Miguel Vatter, profesor de la Universidad de Flinders (Australia), ofrece un trazado genealógico que vincula la concepción secularizada de dignidad humana en Dante Alighieri, tal y como es interpretada por Kantorowicz, con la idea contemporánea de revolución legal. Miriam Jerade, académica de la UNAM (México), explora afinidades electivas entre Rosenzweig y Derrida en lo que concierne a la formulación de un tipo alternativo de decisión, que no replica el conocido decisionismo soberano de Schmitt. Gabriela Rodríguez Rial, profesora de la Universidad de Buenos Aires (Argentina), discute las implicancias teológico-políticas de las dos últimas partes del Leviatán de Hobbes, en general desfavorecidas por la crítica, y encuentra nuevos modos de pensar a partir de ellas el vínculo entre lo teológico y lo político en la soberanía moderna. Rodrigo Karmy, académico de la Universidad de Chile (Chile), plantea tensiones al interior de la teología política musulmana y sugiere que la teoría feminista musulmana contemporánea puede ser leída como un retorno al gesto de Averroes de cuestionar la apropiación --teológica y patriarcal-- del mensaje universal del Corán. Ely Orrego, doctoranda de la Universidad de Northwestern (Estados Unidos), aborda la posibilidad de pensar una teología política vinculada a la crítica eco-feminista y a perspectivas de-coloniales situadas en, y desde, América Latina. Emmanuel Taub, investigador del CONICET (Argentina), explora la espe-

${ }^{11}$ Ejemplos de la madurez de esa recepción son, entre otros muchos posibles, el número especial de la Revista Pléyade (N. 8 de 2011) dedicado exclusivamente a la teología política y la revista Deus Mortalis, dirigida por Jorge Dotti hasta su muerte en 2018, en la que se han publicado numerosos artículos científicos sobre esta temática. 
cificidad de la experiencia de lo secular en el judaísmo como una manera de desplazar concepciones sedimentadas de lo secular y de lo antropomórfico de cuño predominantemente cristiano. Por último, James Martel, académico de la San Francisco State University (Estados Unidos), discute a Benjamin y Nietzsche buscando rescatar a partir de ellos las condiciones de posibilidad del ateísmo y exponer las fuentes teológicas ocultas de nuestros marcos legales y económicos vigentes.

Por último, cabe decir que la teología política es en nuestros días un espacio académico que cultiva la reflexividad frente a los dogmatismos, tanto religiosos como secularizantes, así como el interés sistemático por abordar y comprender los nuevos modos de darse de lo religioso en lo secular y de lo secular en lo religioso. Esperamos que este dossier pueda hacer una contribución seria y duradera a estos objetivos que nos hemos propuesto, a sabiendas de que resulta doblemente difícil participar en igualdad de condiciones en una discusión académica global que todavía avanza lentamente en advertir y visibilizar las iniciativas y propuestas filosóficas que surgen desde el sur global. 


\section{BIBLIOGRAFIA}

Adorno, Theodor y Max Horkheimer, Dialectic of Enlightenment: Philosophical Fragments. Palo Alto: Stanford, Stanford University Press, 2007.

Agamben, Giorgio, Homo Sacer: Sovereign Power and Bare Life. Palo Alto: Stanford University Press, 1995.

, State of Exception, translated by Kevin Attell. Chicago: University of Chicago Press, 2005.

, The Kingdom and the Glory: for a Theological Genealogy of Economy and Government. Palo Alto: Stanford University Press, 2011.

Asad, Talal, Formations of the Secular: Christianity, Islam, Modernity. Palo Alto: Stanford U. Press, 2003.

, Secular Translations: Nation-State, Modern Self and Calculative Reason. New York: Columbia University Press, 2018.

Assmann, Jan, Of God and Gods: Egypt, Israel, and the Rise of Monotheism. Madison: University of Wisconsin Press, 2008. , The Invention of Religion. Princeton: Princeton University Press, 2018.

Benjamin, Walter, "Critique of Violence", trans. Edmund Jephcott, eds Marcus Bullock and Michael Jennings, en Selected Writings Volume 1. Cambridge: Harvard University Press, 1999: 236-252.

Bluemenberg, Hans, The Legitimacy of the Modern Age. Boston: MIT Press, 1985.

Casanova, José, Public Religion in the Modern World. Chicago: University of Chicago Press, 1994.

Cheng Patrick, Radical Love: An Introduction to Queer Theology. New York: Seabury Books, 2001.

Clooney, Francis, “Restoring 'Hindu Theology' as a Category in Indian Intellectual Discourse" en Gavin Flood (ed.) The Blackwell Companion to Hinduism. Oxford: Blackwell Publishing, 2003, pp. 447-477.

Connolly, William, The Ethos of Pluralization, Minneapolis: University of Minnesota Press: 1995.

, Why I am not a Secularist? Minneapolis: University of Minnesota Press, 2000.

Dean-Drummond, Celia y David Clough, Creaturely Theology: On God, Humans, and other Animals (London: SCM Press, 2009).

Derrida, Jacques, Acts of Religion. London: Routledge, 2001.

Dotti, Jorge, “Teología política y excepción”, Daimon Revista Internacional de Filosofia, (13): 129-140.

, “Quién mató al Leviatán? Schmitt intérprete de Hobbes en el contexto del nacionalsocialismo", Deus Mortalis, Nro 1, 2002: 93-190.

Esposito, Roberto, Two: The Machine of Political Theology and the Place of Thought. New York: Fordham, 2015. 
Fenves, Peter, The Messianic Reduction: Walter Benjamin and the Shape of Time. Palo Alto: Stanford University Press, 2010.

Girard, René, Violence and the Sacred. Baltimore: Johns Hopkins University Press, 1979.

Habermas, Jürgen, Europe: The Faltering Project. Malden: Polity Press, 2009.

Hägglund, Martin, This Life: Secular Faith and Spiritual Freedom. New York: Pantheon Books, 2019.

Hammill, Graham y Julia Lupton, Political Theology \& Early Modernity. Chicago:

University of Chicago Press, 2012.

Jaspers, Karl, "The Axial Period" en Karl Jaspers, The Origin and Goal of History. New Haven: Yale University Press, 1953: 1-25.

Kahn, Victoria, The Future of Illusion: Political Theology and Early Modern Texts. $\quad$ Chicago: University of Chicago Press, 2016.

Kantorowicz, Ernst H., The King's Two Bodies: A Study in Mediaeval Political Theology. Princeton: Princeton University Press, 1997.

Keller, Catherine y Mary-Jane Rubenstein (eds.), Entangled Worlds: Religion, Science, and New Materialisms. New York: Fordham University Press, 2017.

Laclau, Ernesto, "On the Names of God", en Hent De Vries y Lawrence Sullivan (eds.), Political Theologies: Public Religions in a Post-Secular World. New York: Fordham University Press, 2006, pp. 137-147.

Lefort, Claude, “The Permanence of the Theologico-Political?" en Hent De Vries y Lawrence Sullivan (eds.), Political Theologies: Public Religions in a Post-Secular World. New York: Fordham University Press, 2006, pp. 148187.

Linzey, Andrew, Animal Theology. Champaign: University of Illinois Press, 1994.

, Creatures of the Same God: Explorations in Animal Theology. Brooklyn: Lantern Books, 2009.

Löwith, Karl, Meaning in History: The Theological Implications of the Philosophy of History. Chicago: University of Chicago Press, 1957.

Lupton, Julia Reinhart, Citizen Saints: Shakespeare and Political Theology. Chicago: University of Chicago Press, 2005.

Mahmood, Saba, Politics of Piety: The Islamic Revival and the Feminist Subject. Princeton: Princeton University Press, 2011.

, Religious Difference in a Secular Age: A Minority Report. Princeton: Princeton University Press, 2015.

Maritain, Jacques, El hombre y el estado. Madrid: Encuentro, 2002.

Martel, James, The One and Only Law: Walter Benjamin and the Second Commandment. Anne Arbor: University of Michigan Press, 2014.

Meier, Heinrich, Leo Strauss and the Theologico-Political Problem. Cambridge: Cambridge University Press, 2006.

Meyer, Eric Daryl, Inner Animalities: Theology and the End of the Human. New York: Fordham University Press, 2018. 
Moore, Stephen D. (ed.), Divinanimality: Animal Theory, Creaturely Theology. New York: Fordham University Press, 20014.

Peterson, Erik, El Monoteísmo como problema politico. Madrid: Trotta, 1999.

Rosenzweig, Franz, The Star of Redemption. Madison: University of Wisconsin Press, 2005.

Santner, Eric, On the Psychotheology of Everyday Life. Chicago: University of Chicago Press, 2001.

, On Creaturely Life: Rilke, Benjamin, Sebald. Chicago: University of Chicago Press, 2006.

, The Royal Remains: The People's Two Bodies and the Endgames of Sovereignty. Chicago: University of Chicago Press, 2011.

Schmitt, Carl, Political Theology: Four Chapters on the Concept of Sovereignty, Chicago: Chicago University Press, 2006.

Schwartz, Regina, Sacramental Poetics at the Dawn of Secularism: When God Left the World. Palo Alto: Stanford, 2008.

Singh, Aakash, "The Political Theology of Navarayana Buddhism" en Péter Losonczi, Mika Luoma-aho y Aakash Singh (eds.) The Future of Political Theology: Religious and Theological Perspectives. London: Routledge, 2012: pp. 159-172

Spinoza, Baruch, Theological-Political Treatise, translated by Samuel Shirley. Indianapolis: Hackett Publishing Company, 2001.

Strauss, Leo, Spinoza 's Critique of Religion. Chicago: Chicago University Press, 1997.

Warner, Michael, Jonathan Vanantwerpen y Craig Calhoun, Varieties of Secularism in a Secular Age, Cambridge: Harvard University Press, 2013.

Weber, Max, The Protestant Ethic and the Spirit of Capitalism, translated by Talcott Parsons, NY: Charles Scribner's Sons, 1958.

Zizek, Slavoj y John Millbank, The Monstrosity of Christ: Paradox or Dialectic? Creston David (ed.) Boston: The MIT Press, 2011. 